PROCEEDINGS OF THE AMERICAN MATHEMATICAL SOCIETY

Volume 124, Number 6, June 1996

\title{
ON CLOSE TO LINEAR COCYCLES
}

\author{
H. B. KEYNES, N. G. MARKLEY, AND M. SEARS \\ (Communicated by Linda Keen)
}

\begin{abstract}
If we have a flow $\left(X, \mathbb{Z}^{m}\right)$ and a cocycle $h$ on this flow, $h: X \times$ $\mathbb{Z}^{m} \rightarrow \mathbb{R}^{m}$, then $h$ is called close to linear if $h$ can be written as the direct sum of a linear (constant) cocycle and a cocycle in the closure of the coboundaries. Many of the desirable consequences of linearity hold for such cocycles and, in fact, a close to linear cocycle is cohomologous to a cocycle which is norm close to a linear one. Furthermore in the uniquely ergodic case all cocycles are close to linear. We also establish that a close to linear cocycle which is covering is cohomologous to one with the special property that it can be extended by piecewise linearity to an invertible cocycle from $X \times \mathbb{R}^{m}$ to itself. This implies that a suspension obtained from a close to linear cocycle is isomorphic to a time change of the suspension obtained from the identity cocycle.
\end{abstract}

\section{The SPACE OF COCYCLES}

This paper is one of a sequence ([2], [1], [3], [4]) designed to understand the structure of the space of continuous cocycles and the suspension flows they can be used to produce. Here we identify and study a particularly well-behaved class which we call "close to linear".

Let $X$ be a compact metric space and let $\mathbb{Z}^{m}$ denote the integer lattice in $\mathbb{R}^{m}, m$ dimensional Euclidean space. We will assume that $\mathbb{Z}^{m}$ acts as a group of commuting homeomorphisms on $X$, that is, we have a flow $\left(X, \mathbb{Z}^{m}\right)$. A cocycle for such a flow is a continuous map $h: X \times \mathbb{Z}^{m} \rightarrow \mathbb{R}^{m}$ such that for all $x \in X, a, b \in \mathbb{Z}^{m}$ we have $h(x, a+b)=h(x, a)+h(a x, b)$ where $a x$ denotes the action of $a$ on $x$. This relationship is called the cocycle equation. Observe that the range of $h$ could be $\mathbb{R}^{n}$ for any $n \geq 1$ and, indeed, by looking at the coordinate functions which are also cocycles we could do analysis by taking $n=1$. (This viewpoint is exploited in [4].) However, as we will see below, the case $n=m$ is the appropriate environment in which to investigate the construction of $\mathbb{R}^{m}$ flows using cocyles and we will restrict ourselves to that situation in this paper.

Let $\mathcal{C}$ denote the set of cocycles on $\left(X, \mathbb{Z}^{m}\right)$. Clearly $\mathcal{C}$ is a vector space over $\mathbb{R}$. Using the norm $|t|=\sum_{i=1}^{m}\left|t_{i}\right|$ for $t=\left(t_{1}, \ldots, t_{m}\right) \in \mathbb{R}^{m}$,

$$
\|h\|=\sup \left\{\frac{|h(x, a)|}{|a|}: x \in X \text { and } a \in \mathbb{Z}^{m}\right\}
$$

defines a norm on $\mathcal{C}$. Using the cocycle equation it is not hard to show that

$$
\|h\|=\sup \left\{\left|h\left(x, e_{j}\right)\right|: x \in X \text { and } 1 \leq j \leq m\right\}
$$

Received by the editors February 25, 1994 and, in revised form, November 11, 1994.

1991 Mathematics Subject Classification. Primary 58F25; Secondary 28D10, 54H20.

(C)1996 American Mathematical Society 
where $e_{1}, \ldots, e_{m}$ is the standard basis for $\mathbb{R}^{m}$. With this norm $\mathcal{C}$ turns out to be a separable Banach space.

There are several easy ways of obtaining cocycles. If $T \in \mathcal{L}$, the linear operators from $\mathbb{R}^{m}$ to itself, then $h(x, a)=T(a)$ defines a cocycle in $\mathcal{C}$. Conversely if $h \in \mathcal{C}$ and $h(x, a)=h(y, a)$ for all $x, y \in X$ and $a \in \mathbb{Z}^{m}$, then the map $a \rightarrow h(x, a)$ is linear. Consequently $\mathcal{L}$ is a closed subspace of $\mathcal{C}$ which is called the space of constant cocyles.

The second easy way to produce cocycles is as follows. Suppose $f$ is a continuous function from $X$ into $\mathbb{R}^{m}$. Define $h \in \mathcal{C}$ by setting $h(x, a)=f(a x)-f(x)$. Such a cocyle is called a coboundary and the coboundaries, $\mathcal{B}$, form another subspace of $\mathcal{C}$. If two cocycles differ by a coboundary we will say they are cohomologous. These ideas are important because cohomologous cocycles have essentially the same properties.

Form the closed subspace $\mathcal{D}=\mathcal{L}+\overline{\mathcal{B}}$. It is the subspace $\mathcal{D}$ which is the subject of study of this paper. We refer to elements of $\mathcal{D}$ as "close to linear". In essence $\mathcal{D}$ consists of cocycles cohomologous to linear cocycles (which are essentially trivial) and limits of sequences of such cocycles. We want to understand the set $\mathcal{D}$; it may be rather rich (see $\S 2$ ), but we will establish that the members of $\mathcal{D}$ are all "wellbehaved" cocycles (see $\S 4$ ). Of course in terms of the cocycle norm, a linear cocycle plus a coboundary may be very far away from a linear one. However, we will show that many consequences of linearity persist in $\mathcal{D}$. Furthermore it will turn out that every element of $\mathcal{D}$ is cohomologous to a cocycle which is close to a linear one in the norm sense. These ideas will justify the terminology.

Cocycles are important tools in the construction of certain special $\mathbb{R}^{m}$ flows, namely the $\mathbb{R}^{m}$ suspensions. So assume we have a flow $\left(X, \mathbb{Z}^{m}\right)$ and a cocycle $h$. Form the space $X \times \mathbb{R}^{m}$ and note that there is a trivial $\mathbb{R}^{m}$ action on this space given by $(x, t) s=(x, t+s)$. Also for each $a \in \mathbb{Z}^{m}$, define a homeomorphism $T_{a}: X \times \mathbb{R}^{m} \rightarrow X \times \mathbb{R}^{m}$ by $T_{a}(x, t)=(a x, t-h(x, a))$. It is obvious that each $T_{a}$ commutes with the $\mathbb{R}^{m}$ action and, in fact, the group of these maps gives a $\mathbb{Z}^{m}$ action on $X \times \mathbb{R}^{m}$ because $h$ satisfies the cocycle equation. We now form the quotient space

$$
X_{h}=X \times \mathbb{R}^{m} /\left\{T_{a}: a \in \mathbb{Z}^{m}\right\}
$$

and let $\pi$ be the canonical projection from $X \times \mathbb{R}^{m}$ to $X_{h}$. We thus obtain an $\mathbb{R}^{m}$ flow $\left(X_{h}, \mathbb{R}^{m}\right)$ which we call the $\mathbb{R}^{m}$ suspension of $X$ given by $h$. In the case $m=1$ this construction is just the usual flow under a function since in one dimension every cocycle is given by $h(x, n)=\sum_{i=0}^{n-1} f(i x)$ for $n>0$ and a similar formula for $n<0$, where $f$ is a continuous function. It is easy to check that $X_{h}$ is a compact Hausdorff space in the case where $f>0$, but even in the general one-dimensional case, it is not clear that $X_{h}$ is well behaved in a topological sense or that $X$ is embedded as a global section in the flow. The relationship between properties of $h$ and the corresponding suspension flow $\left(X_{h}, \mathbb{R}^{m}\right)$ was investigated in detail in [1]. The main results we will need from that paper are restated here for completeness.

Definition 1. A cocycle $h \in \mathcal{C}$ is called covering if

(a) $X_{h}$ is a Hausdorff space,

(b) $\pi$ is a local homeomorphism.

If in addition $\pi$ is one-to-one on $X \times\{0\}$, then $h$ is called embedding. 
Note that when $h$ is an embedding cocycle, $X$ is naturally embedded in $X_{h}$ as a global section.

Theorem 1. Suppose $\left(X, \mathbb{Z}^{m}\right)$ has a free dense orbit. A cocycle $h$ is covering if and only if $|h(x, a)| \rightarrow \infty$ uniformly in $x$ as $|a| \rightarrow \infty$.

This theorem is used extensively in what follows. We thus will impose the Standing Assumption that $\left(X, \mathbb{Z}^{m}\right)$ has a free dense orbit.

Theorem 2. If $h: X \times \mathbb{Z}^{m} \rightarrow \mathbb{R}^{m}$ is covering, then $X_{h}$ is a compact metric space. Moreover, there are constants $M_{1}$ and $M_{2}$ such that $M_{1}|a| \leq|h(x, a)| \leq M_{2}|a|$ for all $a \in \mathbb{Z}^{m}$ with $|a|$ sufficiently large.

In fact if $h$ is a covering cocycle into a space $\mathbb{R}^{n}$ and $X_{h}$ turns out to be compact, then $n$ must be equal to $m$. This justifies our restriction to $n=m$.

It is thus clear that the covering cocycles from $X \times \mathbb{Z}^{m}$ to $\mathbb{R}^{m}$ play a key role in constructing well-behaved $\mathbb{R}^{m}$ suspension actions. Furthermore the suspension flows are important in understanding general minimal $\mathbb{R}^{m}$ actions. (See [3].)

As an easy consequence of Theorem 1, we have:

Remark 1. If $L \in \mathcal{L}$, then $L$ is covering if and only if $L$ is invertible as a linear map.

Remark 2. If two covering cocycles are cohomologous, then the corresponding suspensions are isomorphic as flows. Any cocycle cohomologous to a covering cocycle is covering.

Note that by Remark $2, \mathcal{L}+\mathcal{B}$ generates the same suspension flows as $\mathcal{L}$ itself. We want to understand what new suspension flows come from the closure points of $\mathcal{L}+\mathcal{B}$ in $\mathcal{C}$.

Covering cocycles may exhibit a stronger property which is closely related to the structure of the phase space and orbits of the corresponding suspension. This involves extending $h$ from $X \times \mathbb{Z}^{m}$ to $X \times \mathbb{R}^{m}$.

Let $I^{m}=[0,1]^{m}$ be the unit cube in $\mathbb{R}^{m}$. By the standard triangulation of $I^{m}$ we mean the complex $K$ with $|K|=I^{m}$ consisting of the $m$-simplices

$$
\mathcal{S}_{\sigma}=\left\{0, e_{\sigma 1}, e_{\sigma 1}+e_{\sigma 2}, \ldots, e_{\sigma 1}+\ldots+e_{\sigma m}\right\}
$$

and all their faces generated by all permutations $\sigma$ of $(1, \ldots, m)$. It is easy to see that

$$
\left|\mathcal{S}_{\sigma}\right|=\left\{t \in I^{m}: 1 \geq t_{\sigma 1} \geq t_{\sigma 2} \geq \ldots \geq t_{\sigma m} \geq 0\right\} .
$$

Now for each $x \in X, h(x, \cdot)$ can be extended to a map $H(x, \cdot): \mathbb{R}^{m} \rightarrow \mathbb{R}^{m}$. To do this give $\mathbb{R}^{m}$ a simplicial structure with $K+\mathbb{Z}^{m}$ and view $h(x, \cdot)$ as a vertex map into $\mathbb{R}^{m}$. Then let $H(x, \cdot)$ be the piecewise-linear extension of $h(x, \cdot)$ on $\left|K+\mathbb{Z}^{m}\right|=\mathbb{R}^{m}$ to $\mathbb{R}^{m}$. It is clear that $H$ is a jointly continuous map and $H(x, t+a)=h(x, a)+$ $H(a x, t)$ where $t \in \mathbb{R}^{m}$ and $a \in \mathbb{Z}^{m}$.

If $H(x, \cdot)$ is one-to-one and onto for all $x \in X$ the cocycle $h$ is invertible in the sense of [2] where they were called "cocycles for suspensions". If $h$ is invertible, then there is an orbit-preserving homeomorphism from $X_{h}$ onto $X_{i d}$ (the suspension corresponding to the identity map on $\mathbb{Z}^{m}$ ). (Flows with this property are called conjugate.) So the space $X_{h}$ is topologically independent of $h$ and the dynamics differ only in a time change - the orbits are the same as those of $X_{i d}$. In fact $h$ is invertible in the sense that $H(x, \theta(x, t))=t$ where $\theta(x, t)$ is the required 
(continuous) time change. Note that in view of Remark 2 all this holds if $h$ is merely cohomologous to an invertible cocycle.

We first establish that $H$ being onto holds for all covering cocycles.

Proposition 1. If $h$ is covering, then $H(x, \cdot)$ is onto $\mathbb{R}^{m}$ for all $x \in X$.

Proof. Fix $x$ and denote $H(x, \cdot)$ by $H$. If $h$ is covering, $H$ can be extended continuously to an $m$-dimensional sphere by defining $H(x, \infty)=\infty$. Now if $H$ is not onto, then $H$ can be thought of as a map from $S_{r}^{m}$ to $\mathbb{R}^{m}$ (denoted by $H$ again) where $S_{r}^{m}$ is the $m$-sphere of radius $r$ and $r$ is chosen so large that $|H(x, t)-H(x, s)| \geq 1$ for $|t-s| \geq r$. (That this can be done is a consequence of the fact that $|h(x, a)| \geq M_{1}|a|$ for $a$ sufficiently large.) By the Borsuk-Ulam theorem, we now have a pair of antipodal points $s$ and $-s$ say, with $H(x, s)=H(x,-s)$. But $|s-(-s)|=2 r$ which is a contradiction.

Thus if $h$ is covering and $H$ is one-to-one at each $x \in X$, then $h$ is invertible. Furthermore the extension of $h$ is piecewise-linear in the sense of the discussion above. If $h$ is covering and $H$ is one-to-one for all $x \in X$, then we call $h$ a piecewiselinear invertible cocycle ( $\mathrm{P}-\mathrm{L}$ invertible).

The following sections will bring these concepts together in a natural way for $\mathcal{D}=\mathcal{L}+\overline{\mathcal{B}}$. In $\S 2$ we will note that $\mathcal{D}$ is normally a rich subspace of $\mathcal{C}$ worthy of study in its own right. In $\S 3$ we will establish that an element of $\mathcal{D}$ has a close relationship to a linear cocycle motivating the term "close to linear" for its elements. We also establish that if $\left(X, \mathbb{Z}^{m}\right)$ is uniquely ergodic (i.e. there is only one probability measure on $X$ which is invariant under $\left.\mathbb{Z}^{m}\right)$, then in fact $\mathcal{D}$ is all of $\mathcal{C}$ so that in this case all the results apply to all cocycles. Finally in $\S 4$ we will show that each covering cocycle in $\mathcal{D}$ is cohomologous to a P-L invertible cocycle so that the suspensions obtained from members of $\mathcal{D}$ are all conjugate to the identity suspension.

\section{The subspace $\mathcal{D}=\mathcal{L} \oplus \overline{\mathcal{B}}$}

We first establish that $\mathcal{D}$ is actually a direct sum.

Proposition 2. $\mathcal{D}=\mathcal{L} \oplus \overline{\mathcal{B}}$.

Proof. Suppose $L \in \mathcal{L} \cap \overline{\mathcal{B}}$ and $L \neq 0$. Choose a unit vector $v$ such that $L v \neq 0$ and find a sequence $\left\{a_{k}\right\}$ in $\mathbb{Z}^{m}$ with $\left|a_{k}-k v\right| \leq m$. In this case $\left|L a_{k}\right| \geq B k$ for some $B>0$ and $k$ large enough. Now choose $f \in \mathcal{B}$ such that $\|L-f\|<B / 2$. Then $(B / 2)\left|a_{k}\right| \geq\left|L a_{k}-f\left(x, a_{k}\right)\right| \geq\left|L a_{k}\right|-\left|f\left(x, a_{k}\right)\right| \geq B k-C$ where $C$ is some bound for $f$. Thus $C \geq B k-(B / 2)\left|a_{k}\right|=(B / 2) k+(B / 2)\left(k|v|-\left|a_{k}\right|\right) \geq(B / 2) k-(B / 2) m$. Letting $k \rightarrow \infty$ gives a contradiction.

In view of Proposition 2, if $h \in \mathcal{D}$ we can associate with $h$ a fixed $L_{h} \in \mathcal{L}$ and $f_{h} \in \overline{\mathcal{B}}$ so that $h$ has the (unique) decomposition $h=L_{h}+f_{h}$. We immediately obtain $\overline{\mathcal{B}}=\left\{h \in \mathcal{D}: L_{h}=0\right\}$.

The following lemma is helpful in dealing with elements of $\overline{\mathcal{B}}$.

Lemma 1. If $f \in \overline{\mathcal{B}}$, then $|f(x, a)| /|a| \rightarrow 0$ uniformly in $x$ as $|a| \rightarrow \infty$.

Proof. Given $\varepsilon>0$, let $g \in \mathcal{B}$ with $\|g-f\|<\varepsilon / 2$. As $g$ is bounded, choose $A>0$ so that $|g(x, a)| /|a|<\varepsilon / 2$ when $|a| \geq A$. Now $|f(x, a)| \leq|f(x, a)-g(x, a)|+|g(x, a)|$ and so $|f(x, a)| /|a|<\varepsilon$ whenever $|a| \geq A$. 
Corollary 1. If $h \in \mathcal{D}$, then $\left|h(x, a)-L_{h}(a)\right| /|a| \rightarrow 0$ uniformly in $x$ as $|a| \rightarrow \infty$.

We now turn to the situation for covering cocycles.

Proposition 3. If $h$ is covering, then $h+\overline{\mathcal{B}}$ consists of covering cocycles.

Proof. As $h$ is covering, $|h(x, a)| \geq M_{1}|a|$ for some $M_{1}>0$ and $a$ with $|a|$ sufficiently large. If $f \in \overline{\mathcal{B}}$, then $|h(x, a)+f(x, a)| /|a| \geq|h(x, a)| /|a|-|f(x, a)| /|a| \geq M_{1} / 2$ for $|a|$ sufficiently large by Lemma 1 .

Corollary 2. If $h \in \mathcal{D}$, then $h$ is covering if and only if $L_{h}$ is invertible.

Proof. If $h$ is covering, then $|h(x, a)| /|a| \geq M_{1}$ for $|a|$ large enough and $L_{h}$ must be invertible by Corollary 1 . The converse follows from the proposition and Remark 1.

Theorem 3. Suppose $\mathcal{B}$ is not closed. Then $\mathcal{C}$ contains non-trivial covering cocycles (i.e. covering cocycles not cohomologous to constant ones) and these are dense in $\mathcal{D}$.

Proof. Suppose $f \in \overline{\mathcal{B}}-\mathcal{B}$ and $L \in \mathcal{L}$ is invertible. Then $L+f$ is covering by Corollary 2. If $L+f$ is cohomologous to a constant cocycle $M$ say, then $L+f=M+g$ where $g$ is a coboundary; so by Proposition $2, f=g \in \mathcal{B}$, which is a contradiction. Now if $h \in \mathcal{D}$, then $h=L_{h}+f_{h}$. Then $L^{\prime}+f_{h}$ can be made as close to $h$ as we like with $L^{\prime}$ invertible since the invertible linear maps are dense in $\mathcal{L}$. Now if $f_{h} \in \mathcal{B}$ and we choose $f \in \overline{\mathcal{B}}-\mathcal{B}$ with $\|f\|$ sufficiently small, then $f_{h}+f \notin \mathcal{B}$ and $L^{\prime}+f_{h}+f$ is arbitrarily close to $h$.

We show in [4] that under our standing assumption of a free dense orbit, $\overline{\mathcal{B}} \neq$ $\mathcal{B}$ for real-valued cocycles. Of course this applies to the situation here also by considering the coordinate maps. Thus, in fact, the supposition that $\mathcal{B}$ is not closed is unnecessary in the statement of the theorem provided $\left(X, \mathbb{Z}^{m}\right)$ has a free dense orbit.

Proposition 4. The covering cocycles are open in $\mathcal{C}$.

Proof. This is an easy consequence of the definition of the norm and the fact that a cocycle is covering if and only if $|h(x, a)| \geq M_{1}|a|$ for some $M_{1}>0$ and $|a|$ sufficiently large.

Corollary 3. The covering cocycles form an open dense set in $\mathcal{D}$.

\section{LineARisAtion IN $\mathcal{D}$}

The next result indicates a precise sense in which $h \in \mathcal{D}$ is "close to linear".

Theorem 4. Let $h \in \mathcal{C}$. The following are equivalent:

(a) $h \in \mathcal{D}$.

(b) There is a linear cocycle $L \in \mathcal{L}$ such that we can find a sequence of cocycles $\left\{h_{n}\right\}$ all cohomologous to $h$ with $h_{n} \rightarrow L$.

(c) The distance between the sets $\mathcal{L}$ and $h+\mathcal{B}$ is 0 .

Proof. (a) $\Rightarrow$ (b). If $h \in \mathcal{D}$, let $f_{n} \in \mathcal{B}$ be a sequence with $f_{n} \rightarrow f_{h}$. Now $h-f_{n} \rightarrow L_{h}$ and $h-f_{n}$ is cohomologous to $h$.

(b) $\Rightarrow(\mathrm{c})$. Obvious. 
(c) $\Rightarrow$ (a). If $d(h+\mathcal{B}, \mathcal{L})=0$, then there exist $f_{n} \in \mathcal{B}$ and $L_{n} \in \mathcal{L}$ such that $\left\|h+f_{n}-L_{n}\right\| \leq 1 / n$. Thus $f_{n}-L_{n}$ is a sequence in $\mathcal{D}$ converging to $-h$. So $h \in \overline{\mathcal{D}}=\mathcal{D}$.

Given $h \in \mathcal{C}$, there is a natural way of constructing cohomologous cocycles which average the values of $h$ over a central portion of each orbit. Choose $\ell$ to be a positive integer and define $P_{\ell}(x)=\left(1 / \ell^{m}\right) \sum h(x, a)$ where the sum is taken over all $a \in \mathbb{Z}^{m}$ such that $1 \leq a_{j} \leq \ell$ for all $1 \leq j \leq m$. Since $P_{\ell}$ is continuous, we can use it to define a coboundary and set $h_{\ell}(x, a)=P_{\ell}(a x)-P_{\ell}(x)+h(x, a)$. Thus $h_{\ell}$ is cohomologous to $h$. Now

$$
\begin{aligned}
h_{\ell}\left(x, e_{i}\right) & =h\left(x, e_{i}\right)+\frac{1}{\ell^{m}} \sum\left\{h\left(e_{i} x, a\right)-h(x, a): 1 \leq a_{j} \leq \ell, 1 \leq j \leq m\right\} \\
& =h\left(x, e_{i}\right)+\frac{1}{\ell^{m}} \sum\left\{h\left(a x, e_{i}\right)-h\left(x, e_{i}\right): 1 \leq a_{j} \leq \ell, 1 \leq j \leq m\right\} \\
& =\frac{1}{\ell^{m}} \sum\left\{h\left(a x, e_{i}\right): 1 \leq a_{j} \leq \ell, 1 \leq j \leq m\right\} .
\end{aligned}
$$

This argument to construct the cohomologous cocycles was shown to us by $\mathrm{H}$ Furstenberg.

In the one-dimensional case it can be shown that the sign of $\int h(x, 1) d \mu$ is the same for every invariant measure $\mu$, say positive. (See Theorem 1.12 and its proof in [1].) If $h_{\ell}(x, 1)$ is not eventually strictly positive, the above expansion for $h_{\ell}$ can be used to produce an invariant measure with non-positive integral. Thus in the one-dimensional case a covering cocycle generated by an unrestricted continuous function is cohomologous to one generated by a strictly positive or negative function. Thus for real suspensions one can restrict oneself to that simpler situation without loss of generality.

We can now characterise $\mathcal{D}$ in terms of the integrals of $h$. Define an operator $A_{\ell}^{x}: C(X) \rightarrow \mathbb{R}$ by $A_{\ell}^{x}(f)=\frac{1}{\ell^{m}} \sum f(a x)$ where the sum is over all $a$ with $1 \leq a_{i} \leq \ell$.

Lemma 2. $\int_{X} f(x) d \mu=0$ for all invariant probability measures $\mu$ if and only if $A_{\ell}^{x}(f) \rightarrow 0$ uniformly in $x$ as $\ell \rightarrow \infty$.

Proof. Suppose that $A_{\ell}^{x}(f)$ does not converge to 0 uniformly in $x$ as $\ell \rightarrow \infty$. We then have $\varepsilon>0, \ell_{k} \rightarrow \infty,\left\{x_{k}\right\}$ with $\left|A_{\ell_{k}}^{x_{k}}(f)\right| \geq \varepsilon$ for all $k$. Since these linear operators are all in the unit ball, we can suppose $A_{\ell_{k}}^{x_{k}}$ converges weakly to a linear operator $\theta$, say. It is easy to check that $\theta$ is invariant in the sense that $\theta(a g)=\theta(g)$ $\left(a \in \mathbb{Z}^{m}\right)$ where $a g(x)=g(a x)$. So $\theta$ is represented by an invariant probability measure $\mu$ and $\int_{X} f(x) d \mu \neq 0$.

The converse is immediate because $A_{\ell}^{x}(f)$ is an ergodic average of $f$.

Theorem 5. Let $h \in \mathcal{C}$ and $\mu$ be an invariant probability measure on $\left(X, \mathbb{Z}^{m}\right)$, and define the linear map $L_{\mu}(a)=\int_{X} h(x, a) d \mu$. Then $h \in \mathcal{D}$ if and only if the maps $L_{\mu}$ are all the same, i.e. the integral of $h$ does not depend on the choice of $\mu$.

Proof. Let $h \in \mathcal{D}$ and fix $a \in \mathbb{Z}^{m}$. By Corollary 1

$$
\left|\frac{1}{k} h(x, k a)-L_{h}(a)\right| \rightarrow 0
$$


uniformly in $x$ as $k \rightarrow \infty$. But by the ergodic theorem applied to the integer action induced by $a$

$$
\frac{1}{k} h(x, k a)=\frac{1}{k} \sum_{j=0}^{k-1} h(j a x, a)
$$

must converge a.e. $\mu$ to an integrable function whose integral is $\int_{X} h(x, a) d \mu$. Thus

$$
L_{h}(a)=\int_{X} L_{h}(a) d \mu=\int_{X} h(x, a) d \mu
$$

and so $L_{\mu}=L_{h}$ for any $\mu$.

Conversely suppose $\int_{X} h(x, a) d \mu=L(a)$ where $L$ is independent of $\mu$. Then by Lemma 2 and the above definition of $h_{\ell}$

$$
\left(h_{\ell}\left(x, e_{i}\right)-L e_{i}\right)_{j}=A_{\ell}^{x}\left(\left(h\left(\cdot, e_{i}\right)-L e_{i}\right)_{j}\right) \rightarrow 0
$$

uniformly in $x$ for any $i, j=1, \ldots, m$. Thus $h_{\ell} \rightarrow L$, and so $h \in \mathcal{D}$ by Theorem 4 .

Corollary 4. If $\left(X, \mathbb{Z}^{m}\right)$ is uniquely ergodic, then $\mathcal{C}=\mathcal{D}$.

The question of when $\mathcal{C}=\mathcal{D}$ in general turns out to be a deeper issue. Note that if $m=1$, unique ergodicity is both necessary and sufficient by Theorem 5 since every continuous function generates a cocycle. The general case is addressed in [4].

\section{P-L invertible Cocycles And $\mathcal{D}$}

We will establish that the P-L invertible cocycles form an open set in $\mathcal{C}$. The results of $\S 3$ then enable us to establish a close relationship between the elements of $\mathcal{D}$ and P-L invertible cocycles.

Lemma 3. Let $N>0$ be an integer and $V=\mathbb{Z}^{m} \cap[-N, N]^{m}$. Suppose $f: V \rightarrow \mathbb{R}^{m}$ and let $F:[-N, N]^{m} \rightarrow \mathbb{R}^{m}$ be the piecewise-linear extension of $f$. Suppose $F$ is one-to-one. There is $\varepsilon>0$ such that if $g: V \rightarrow \mathbb{R}^{m}$ and $\sup _{v \in V}|f(v)-g(v)|<\varepsilon$, then $G$ is one-to-one where $G$ is the piecewise-linear extension of $g$ to $[-N, N]^{m}$.

Proof. Let $\Xi$ be the collection of simplices $\{v+\mathcal{S}: \mathcal{S}$ is in the standard triangulation of $[0,1]^{m}$ and $\left.v \in \mathbb{Z}^{m} \cap[-N, N-1]^{m}\right\}$. If $\mathcal{S} \in \Xi$, then $F$ is an invertible linear map on $\mathcal{S}$ and so $G$ will be invertible on $\mathcal{S}$ also for $g$ close enough to $f$. If $\mathcal{S}_{1}, \mathcal{S}_{2} \in \Xi$ and $\mathcal{S}_{1}$ and $\mathcal{S}_{2}$ are disjoint, we can find a linear function $\gamma: \mathbb{R}^{m} \rightarrow \mathbb{R}$ and $c \in \mathbb{R}$ such that $\gamma(F(w))>c$ for $w \in \mathcal{S}_{1}$ and $\gamma(F(w))<c$ for $w \in \mathcal{S}_{2}$. If $g$ is close enough to $f$ we can ensure $\gamma(G(w))>c$ for $w \in \mathcal{S}_{1}$ and $\gamma(G(w))<c$ for $w \in \mathcal{S}_{2}$. Thus $G\left(\mathcal{S}_{1}\right) \cap G\left(\mathcal{S}_{2}\right)=\phi$.

Now suppose $\mathcal{S}_{1}, \mathcal{S}_{2} \in \Xi$ with a common face $\mathcal{S}^{\prime}$ and assume $\mathcal{S}^{\prime}$ is a largest common face in terms of dimension. Let $v_{1}, \ldots, v_{p}$ be the vertices of $\mathcal{S}^{\prime}$ and then $f\left(v_{1}\right), \ldots, f\left(v_{p}\right)$ are the vertices of $F\left(\mathcal{S}^{\prime}\right)$ which is a largest common face of $F\left(\mathcal{S}_{1}\right)$ and $F\left(\mathcal{S}_{2}\right)$. (Note that $F\left(\mathcal{S}_{1}\right), F\left(\mathcal{S}_{2}\right)$ and $F\left(\mathcal{S}^{\prime}\right)$ are all simplices of the same dimension as $\mathcal{S}_{1}, \mathcal{S}_{2}$ and $\mathcal{S}^{\prime}$ respectively as $F$ is one-to-one.) Now let $\mathcal{H}$ be a hyperplane separating $F\left(\mathcal{S}_{1}\right)$ and $F\left(\mathcal{S}_{2}\right)$ and containing $F\left(\mathcal{S}^{\prime}\right)$.

We can choose $b_{p+1}, \ldots, b_{m} \in \mathcal{H}$ such that if

$$
\gamma(w)=\operatorname{det}\left(w, f\left(v_{2}\right)-f\left(v_{1}\right), \ldots, b_{m}-f\left(v_{1}\right)\right),
$$

then

$$
\mathcal{H}=\{w: \gamma(w)=c\}
$$


where $c=\gamma\left(f\left(v_{1}\right)\right)$. Now for vertices of $\mathcal{S}_{1}$ not in $\mathcal{S}^{\prime}, \gamma$ will be less than $c$ (say), and then for vertices of $\mathcal{S}_{2}$ not in $\mathcal{S}^{\prime}$, it will be greater than $c$. Now for $g$ close enough to $f$, the function $\operatorname{det}\left(w, g\left(v_{2}\right)-g\left(v_{1}\right), \ldots, b_{m}-g\left(v_{1}\right)\right)$ will have the same property because the determinant depends continuously on its arguments. Thus $G\left(\mathcal{S}_{1}\right) \cap G\left(\mathcal{S}_{2}\right)=G\left(\mathcal{S}^{\prime}\right)$.

Since $\Xi$ is finite, this completes the proof.

Theorem 6. The P-L invertible cocycles are open in $\mathcal{C}$.

Proof. Let $h$ be P-L invertible and $M_{1}>0$ such that $|h(x, a)| \geq M_{1}|a|$ for $|a|$ sufficiently large. ( $M_{1}$ exists as $h$ is covering.) Let $g \in \mathcal{C}$ and $\|h-g\|<M_{1} / 2$. Note that $\|g\|<M_{1} / 2+\|h\|=\alpha / m$, say. Using the cocycle equation, if $t \in \mathbb{R}^{m}$ and $a \in \mathbb{Z}^{m}$ is the integer part of $t$, then $|G(x, t)-g(x, a)| \leq m\|g\|$ for all $x \in X$. Now there is an integer $N>0$ (independent of $g$ ) such that $|G(x, t)| \geq \alpha$ for any $x \in X$ and $|t| \geq N$. If not, we can find a sequence $g_{n} \in \mathcal{C}$ satisfying $\left\|g_{n}-h\right\|<$ $M_{1} / 2, t_{n} \in \mathbb{R}^{m}$ with $\left|t_{n}\right| \rightarrow \infty$ and $x_{n} \in X$ such that $\left|G_{n}\left(x_{n}, t_{n}\right)\right|<\alpha$. Thus if $a_{n}$ is the integer part of $t_{n}$, then $\left|g_{n}\left(x_{n}, a_{n}\right)\right|<m\left\|g_{n}\right\|+\alpha<2 \alpha$. But now

$$
\begin{aligned}
M_{1}\left|a_{n}\right| / 2 & \geq\left|h\left(x_{n}, a_{n}\right)-g_{n}\left(x_{n}, a_{n}\right)\right| \\
& \geq\left|h\left(x_{n}, a_{n}\right)\right|-\left|g_{n}\left(x_{n}, a_{n}\right)\right| \geq M_{1}\left|a_{n}\right|-m\left\|g_{n}\right\|-\alpha .
\end{aligned}
$$

So $2 \alpha>\alpha+m\left\|g_{n}\right\| \geq M_{1}\left|a_{n}\right| / 2$ which is a contradiction as clearly $\left|a_{n}\right| \rightarrow \infty$.

Now suppose $g$ is not P-L invertible. An application of the cocycle equation shows that we can find $x \in X, s \in I^{m}$ and $t \in \mathbb{R}^{m}$ with $G(x, s)=G(x, t)$. As $|G(x, s)| \leq m\|g\|<\alpha$, we know that $t \in[-N, N]^{m}$.

Using this $N$, the lemma determines $\varepsilon_{x}$ for $h(x, \cdot)$ restricted to $V$ for each $x \in X$. There is $\delta_{x}>0$ such that $d(x, y)<\delta_{x}$ implies $|h(x, a)-h(y, a)|<\varepsilon_{x} / 2$ for $a \in V$. Cover $X$ with balls of radius $\delta_{x}$ about each $x$ and select a finite subcover. Thus we have $x_{1}, x_{2}, \ldots, x_{n}$, say, with

$$
X=\bigcup_{i=1}^{n}\left\{y: d\left(y, x_{i}\right)<\delta_{x_{i}}\right\} .
$$

Let $\varepsilon=\min \left\{\varepsilon_{x_{1}}, \varepsilon_{x_{2}}, \ldots, \varepsilon_{x_{n}}, M_{1}\right\}$ and suppose that $\|g-h\|<\varepsilon / 2 m N$. Now fix $x \in X$ and choose $x_{i}$ with $d\left(x, x_{i}\right)<\delta_{x_{i}}$. Since $H\left(x_{i}, \cdot\right)$ is invertible, it is enough to show that $\left|g(x, a)-h\left(x_{i}, a\right)\right|<\varepsilon_{x_{i}}$ for $a \in V$. But $\left|g(x, a)-h\left(x_{i}, a\right)\right| \leq$ $|g(x, a)-h(x, a)|+\left|h(x, a)-h\left(x_{i}, a\right)\right| \leq\|g-h\||a|+\varepsilon_{x_{i}} / 2<\varepsilon_{x_{i}}$ for $a \in V$ since $|a| \leq m N$.

Corollary 5. Suppose $h \in \mathcal{D}$ and $h$ is covering. Then $h$ is cohomologous to a $P-L$ invertible cocycle.

Proof. This is immediate from Theorem 4, Corollary 2 and the theorem above since $L \in \mathcal{L}$ is $\mathrm{P}$-L invertible precisely if $L$ is invertible in the usual sense.

Corollary 6. Suppose $h \in \mathcal{D}$ and $h$ is covering. Then the suspension $\left(X_{h}, \mathbb{R}^{m}\right)$ is conjugate to the identity suspension $\left(X_{\mathrm{id}}, \mathbb{R}^{m}\right)$, i.e. $\left(X_{h}, \mathbb{R}^{m}\right)$ is isomorphic to a time change of $\left(X_{\mathrm{id}}, \mathbb{R}^{m}\right)$.

Proof. An invertible cocyle is what was termed a "cocycle for a suspension" in [2], so this follows from the above corollary and Theorem 2.2 of [2].

Corollary 7. If $\left(X, \mathbb{Z}^{m}\right)$ is uniquely ergodic, then every $\mathbb{R}^{m}$ suspension of it given by a covering cocycle is isomorphic to a time change of the identity suspension. 
In the case $m=1$, we have already noted that up to coboundaries we can assume $f>0(f<0)$. In this case, of course, $h$ is increasing (decreasing) and so $\mathrm{P}-\mathrm{L}$ invertible. Thus the conclusions of the above theorem and corollaries hold for $m=1$ without any assumption on the size of $\mathcal{D}$.

\section{REFERENCES}

1. H. Furstenberg, H. B. Keynes, N. G. Markley, and M. Sears, Topological Properties of $\mathbb{R}^{n}$ suspensions and growth properties of $\mathbb{Z}^{n}$ cocycles, Proc. London Math. Soc., 66 No 3 (1993), 431-448. MR 94c:58176

2. H. B. Keynes, and M. Sears, Time changes for $\mathbb{R}^{n}$ flows and suspensions, Pacific J Math., 130 No 1 (1987), 97-113.

3. H. B. Keynes, N. G. Markley, and M. Sears, The structure of $\mathbb{R}^{n}$ minimal actions, Quaestiones Mathematicae, 16 No 1 (1993), 81-102. MR 94h:54052

4. H. B. Keynes, N. G. Markley, and M. Sears, Ergodic averages and integrals of cocycles, Acta Math. Univ. Comemanae LXIV (1995), 123-139.

School of Mathematics, University of Minnesota, Minneapolis, Minnesota 55455

E-mail address: keynes@math.umn.edu

Department of Mathematics, University of Maryland, College Park, Maryland 20742

E-mail address: ngm@glve.umd.edu

Department of Mathematics, University of the Witwatersrand, Johannesburg, South AFricA

E-mail address: 036mis@cosmos.wits.ac.za 